\title{
Flexible and transparent capacitive pressure sensor with patterned microstructured composite rubber dielectric for wearable touch keyboard application
}

\author{
Ruilong Shi ${ }^{1,2}$, Zheng Lou ${ }^{1 *}$, Shuai Chen ${ }^{1}$ and Guozhen Shen ${ }^{1,2^{*}}$
}

\begin{abstract}
The development of pressure sensors with highly sensitivity, fast response and facile fabrication technique is desirable for wearable electronics. Here, we successfully fabricated a flexible transparent capacitive pressure sensor based on patterned microstructured silver nanowires (AgNWs)/ polydimethylsiloxane (PDMS) composite dielectrics. Compared with the pure PDMS dielectric layer with planar structures, the patterned microstructured sensor exhibits a higher sensitivity $\left(0.831 \mathrm{kPa}^{-1},<0.5 \mathrm{kPa}\right)$, a lower detection limit, good stability and durability. The enhanced sensing mechanism about the conductive filler content and the patterned microstructures has also been discussed. A $5 \times 5$ sensor array was then fabricated to be used as flexible and transparent wearable touch keyboards systems. The fabricated pressure sensor has great potential in the future electronic skin area.
\end{abstract}

Keywords: silver nanowires, capacitive pressure sensor, flexible, transparent, wearable electronics, e-skin

\section{INTRODUCTION}

Flexible and bendable pressure sensors with high sensing performances have obtained great attention [1-6], due to their broad applications in human-machine interactions $[5,7,8]$, health monitoring $[9,10]$, medical diagnostics $[2,11,12]$ and artificial intelligence [13], etc. Recently, high sensitivity, large-scale and high resolution pressure sensor have made significant breakthrough based on different physical transduction mechanisms, such as piezoelectricity, piezoresistivity and capacitance. Compared with the other types of pressure sensors, capacitive pressure sensors with high accuracy in detecting static loads, low power consumption, low hysteresis and large response ranges, have obtained great success in the field of consumer electronics, including biometric identifica- tion $[13,14]$, touchpads $[7,15]$ and touchscreens.

Due to the transduction mechanism to the applied mechanical force, the sensitivity of the capacitive pressure sensor primarily depends on the distance between the two electrodes and the dielectric constant of the dielectric layer. Therefore, capacitive sensors require a dielectric material that is easy to compress and responds rapidly to pressure applied and released. In addition to using foam as dielectric material to detect moderate pressure, elastomers are also incorporated into capacitive pressure sensors. However, the inherent characteristics of a single dielectric limit the further development of capacitive pressure sensors. Therefore, to increase the sensing performance, some effective methods have been investigated, including doping fillers in insulating elastic dielectrics [16-20], introducing the ordered microstructures to the dielectric [14,20-24], changing the internal microstructure of the dielectric $[15,25]$, and so on. For example, Schwartz et al. [26] reported a flexible capacitive pressure sensor embedded capacitive sensing element with a microstructured elastomer layer which revealed a fast response within a millisecond range and a great mechanical flexibility. However, few reports focus on the synergistic effect of the fillers and microstructures to improve the capacitive sensing performance.

Herein, we present a highly sensitive, flexible and transparent capacitive pressure sensor with the silver nanowires (AgNWs)/polydimethylsiloxane (PDMS) composite dielectric films. To further enhance the sensitivity, patterned pyramid-type microstructures were introduced to the composite film by using a simple and low-cost method. With patterned microstructures and sensitive composite dielectric films, the sensors have high sensitivity in the low voltage range $\left(0.831 \mathrm{kPa}^{-1},<0.5 \mathrm{kPa}\right)$

\footnotetext{
${ }^{1}$ State Key Laboratory of Superlattices and Microstructures, Institution of Semiconductors, Chinese Academy of Sciences, Beijing 100083, China

${ }^{2}$ College of Materials Science and Opto-electronic Technology, University of Chinese Academy of Sciences, Beijing 100029, China

* Corresponding authors (emails: zlou@semi.ac.cn (Lou Z); gzshen@semi.ac.cn (Shen G))
} 
and very low detection limits of $1.4 \mathrm{~Pa}$, as well as ultrafast response times. It also exhibits excellent operational durability and repeatability. In addition, we fabricated a flexible sensor array for wearing flexible touch keyboard, demonstrating that this capacitive pressure sensor has great potential in the application of human-computer interaction and electronic skin.

\section{EXPERIMENTAL SECTION}

\section{Fabrication of micropatterned silicon mould}

$200 \mathrm{~nm} \mathrm{SiO}$, was deposited on a clean (100) Si wafer using plasma enhanced chemical vapor deposition (PECVD). Then the $\mathrm{SiO}_{2}$ oxide layer was photolithographed using a mask plate. The exposed $\mathrm{SiO}_{2}$ patterns was etched by buffered oxide etch (BOE) $\left(\mathrm{NH}_{4} \mathrm{~F}\right.$ : $\mathrm{HF}=6: 1, v / v)$. Next, the substrate was wet etched anisotropically using tetramethylammonium hydroxide (TMAH) solution (20 wt $\%$ TMAH:isopropanol $=5: 1, v / v)$ at $80^{\circ} \mathrm{C}$ under stirring for $60 \mathrm{~min}$. The remaining $\mathrm{SiO}_{2}$ layer was removed by BOE. After washing with deionized (DI) water, the Si master was deposited on $50 \mathrm{~nm} \mathrm{Au}$ to prevent adhesion.

\section{Synthesis process of AgNWs}

AgNWs were synthesized by hydrothermal method. 0.24 g Polyvinylpyrrolidone (PVP) was fully dissolved in $30 \mathrm{~mL}$ ethylene glycol under $65^{\circ} \mathrm{C}$ for $2 \mathrm{~h}$. After the solution was cooled to room temperature, $0.5 \mathrm{~g}$ of $\mathrm{AgNO}_{3}$ was added and stirred until completely dissolved. $7 \mathrm{~g}$ $\mathrm{FeCl}_{3}$ solution $\left(600 \times 10^{-6} \mathrm{~mol} \mathrm{~L}^{-1}\right.$, ethylene glycol $)$ was poured into the above solution and stirred for $3 \mathrm{~min}$. The mixed solution was then immediately transferred to reaction kettle and placed in an oven at $130^{\circ} \mathrm{C}$ for $5 \mathrm{~h}$. Finally, the precipitate was centrifuged at 7,500 rpm for 5 min with ethylene glycol and then centrifuged twice with acetone and ethanol at 3,500 rpm for $5 \mathrm{~min}$, respectively. The obtained AgNWs were dispersed in ethanol at a concentration of $0.3 \mathrm{mg} \mathrm{mL}^{-1}$.

\section{Fabrication of AgNWs/PDMS mixture solution and the micro-structured composite dielectric layers}

First, the PDMS elastomer (Sylgard 184, Dow Corning) and the curing agent were mixed at a mass ratio of 10:1 and stirred for $30 \mathrm{~min}$, and then evacuated for $15 \mathrm{~min}$. The prepared AgNWs were dispersed in ethanol and its concentration was $0.3 \mathrm{mg} \mathrm{mL}^{-1}$.

Thereafter, different proportions of the AgNWs solution were added to $1 \mathrm{~g}$ PDMS mixture and stirred fully with a magnetic bar to obtain the composite solution with different doping ratio. In order to remove ethanol, the resulting solution was centrifuged at $8,000 \mathrm{rpm}$ for $5 \mathrm{~min}$ and removed the supernatant and then the composite solution was stirred for $20 \mathrm{~min}$ and evacuated at $50^{\circ} \mathrm{C}$ for $15 \mathrm{~min}$. Finally, it was spin-coated onto the micropatterned Si mould at $1,000 \mathrm{rpm}$ and cured at $80^{\circ} \mathrm{C}$ for $2 \mathrm{~h}$ before being peeled off.

\section{Characterization}

The scanning electron microscopy (SEM) images were characterized using an FESEM Shimadzu Japan (SSX550). The optical images were captured using a Canon camera. The computer controlled movable stage (Shandu SJY-500V) and force gauge (Shandu SP-5 and SH-500B) were used to apply the static pressure. A home-made force supplied equipment was used to apply dynamic pressure measurements. The electrical characterization of the flexible capacitive pressure sensor was measured with Keithley 4200 under the signal of $1 \mathrm{~V}$ at $2 \mathrm{MHz}$.

\section{Materials}

All the reagents including ethylene glycol (anhydrous, 99.8\%, Aldrich), PVP $\left(M_{\mathrm{w}}=360,000\right.$, Aldrich $)$, ferric chloride (Aldrich), silver nitrate (Aldrich), acetone (AR, 99.5\%), and anhydrous ethanol (AR, 99.5\%), were used without further purification. PDMS used was SYLGARD 184 Silicone Elastomer Base and Curing Agent. The indium tin oxides (ITO)/polyethylene terephthalate (PET) film $(50 \mu \mathrm{m})$ was commercially available.

\section{RESULTS AND DISCUSSION}

The schematic illustrations for the fabrication of micropatterned Si mould and flexible microstructured composite AgNWs/PDMS film were illustrated in Fig. 1. The detailed process of fabrication was described in the experimental section. Briefly, the micropatterned Si mould was first fabricated using conventional photolithography and wet etching (Fig. 1a). The microstructures of the $\mathrm{Si}$ mould were regular and uniform as shown in Fig. S1. Moreover, the mould can be reused for many times, thus the repeatedly operations do not affect the uniformity of the transferred microstructured dielectric films. The prepared AgNWs/PDMS solution was spin-coated on the Si mould, which were then peeled off to obtain the flexible microstructured AgNWs/PDMS composite dielectric film (Fig. 1b). The concentration of AgNWs in ethanol and the detailed fabrication method of AgNWs/PDMS mixture solution can be find in the experimental section. Then we used the as-obtained film to prepare a flexible capacitive pressure sensor. 


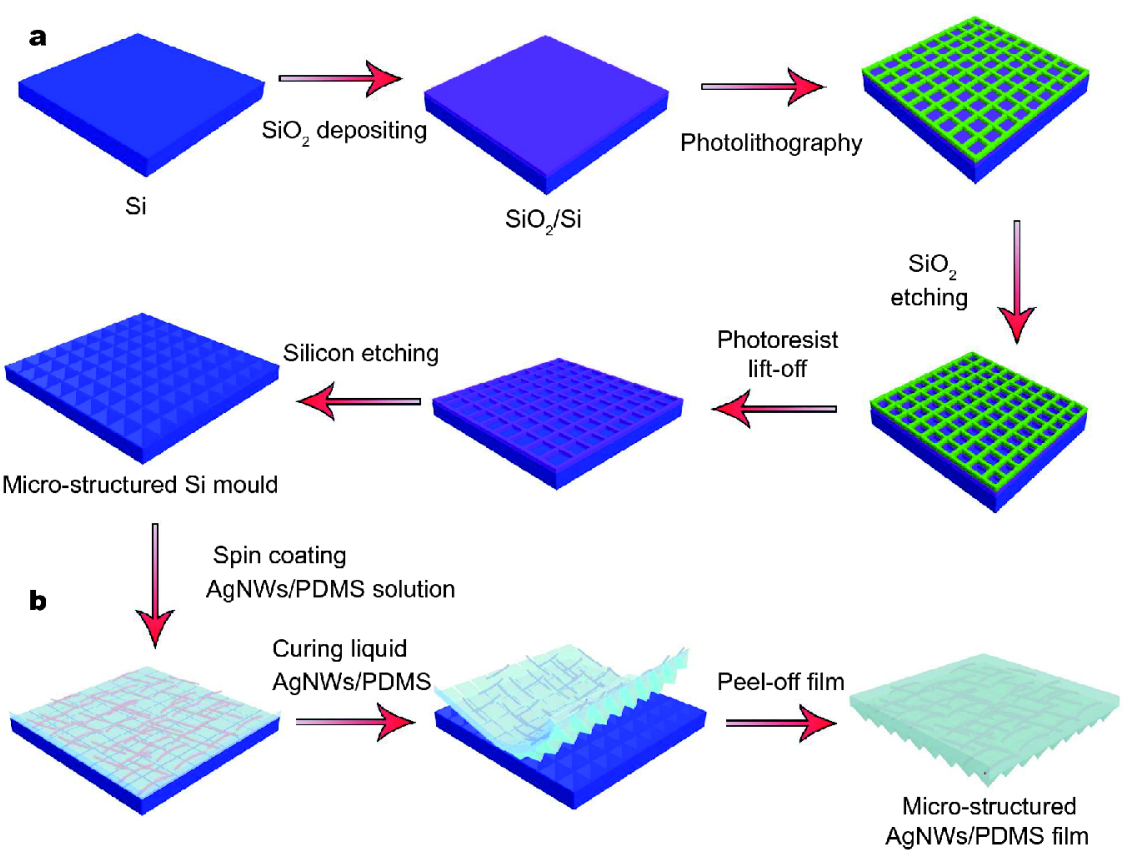

Figure 1 Schematic illustration for the fabrication of (a) micropatterned Si mould and (b) flexible microstructured AgNWs/PDMS composite dielectric film.

Fig. 2a illustrates the device structure of the flexible capacitive pressure sensor using AgNWs/PDMS composite films as the dielectric films in the middle, sandwiched by two ITO/PET $(\sim 50 \mu \mathrm{m})$ films acting as the upper and lower electrodes. As the PET and AgNWs/PDMS films are flexible, the as-made devices are bendable and hence wearable. The optical image of a single pressure sensor device shows that the device has good transparency (Fig. $2 b$ ). The transparency of the composite films can be easily regulated by doping different mass ratio of AgNWs. SEM image shows the as-grown AgNWs with an average length of $150 \mu \mathrm{m}$ and diameter of $100 \mathrm{~nm}$ (Fig. S2). As shown in Fig. 2c, d, the pyramid-shaped microstructures were regularly arranged and had the same dimensions with a length and a height of about $11.5 \mu \mathrm{m}$ and $7.6 \mu \mathrm{m}$, respectively. The thickness of the micro-structured dielectric layer is about $98.8 \mu \mathrm{m}$, as shown in Fig. S3. Fig. 2e shows the optical transmittance of the AgNWs/PDMS films with various mixing ratios of AgNWs. When the mass ratio of AgNWs and PDMS is $0 \%, 0.03 \%, 0.06 \%$ and $0.12 \%$, the transmittance of the AgNWs/PDMS film is 98 , 91,87 and $71 \%$ under $550 \mathrm{~nm}$ light illumination, respectively.

The sensing mechanism of the capacitive pressure sensor based on the AgNWs/PDMS dielectric layer was illustrated in Fig. 3a. The basic sensing mechanism of the traditional capacitive pressure sensing is equivalent to the parallel plate capacitor, and the capacitance value $C$ is as follows: $C=\varepsilon_{0} \varepsilon S / d$ (Equation 1), where $\varepsilon_{0}$ is the vacuum dielectric constant and $\varepsilon$ is the dielectric constant of the composite film, and $S$ is the relative plate area, and $d$ is the distance between the plates. When the sensor is not loaded, the distance between the upper and lower electrodes is fixed leading to a certain initial capacitance value. However, when the force is applied to the pressure sensor, the dielectric is deformed, and the distance between the plates is reduced to $d$. As a result, the capacitance value $C$ becomes large. Thus, a conventional capacitive pressure sensor may provide a significant change in capacitance proportional to the applied pressure.

In addition to the distance $d$, the change in the dielectric constant $\varepsilon$ can also cause a change in the capacitance value $C$. Therefore, we controlled the dielectric constant of the dielectric layer by doping different proportions of AgNWs in the PDMS dielectric to improve the sensitivity and response range. The change of dielectric constant of the AgNWs/PDMS composites under pressure can be interpreted by the Kirkpatrick and Zallen statistical percolation model [18,29-32], which is used to predict the electrical properties of a percolation system with non-interacting randomly dispersed fillers. Here, the capacitance value $C$ is as follows: $C=\varepsilon_{0} \varepsilon_{a} S / d$ (Equation 2) and $\varepsilon_{\mathrm{a}}=\varepsilon_{\mathrm{d}}\left(f_{\mathrm{c}}-f\right)^{-S}, f_{\mathrm{c}}>f$ (Equation 3), where $\varepsilon_{\mathrm{d}}$ is the effective 

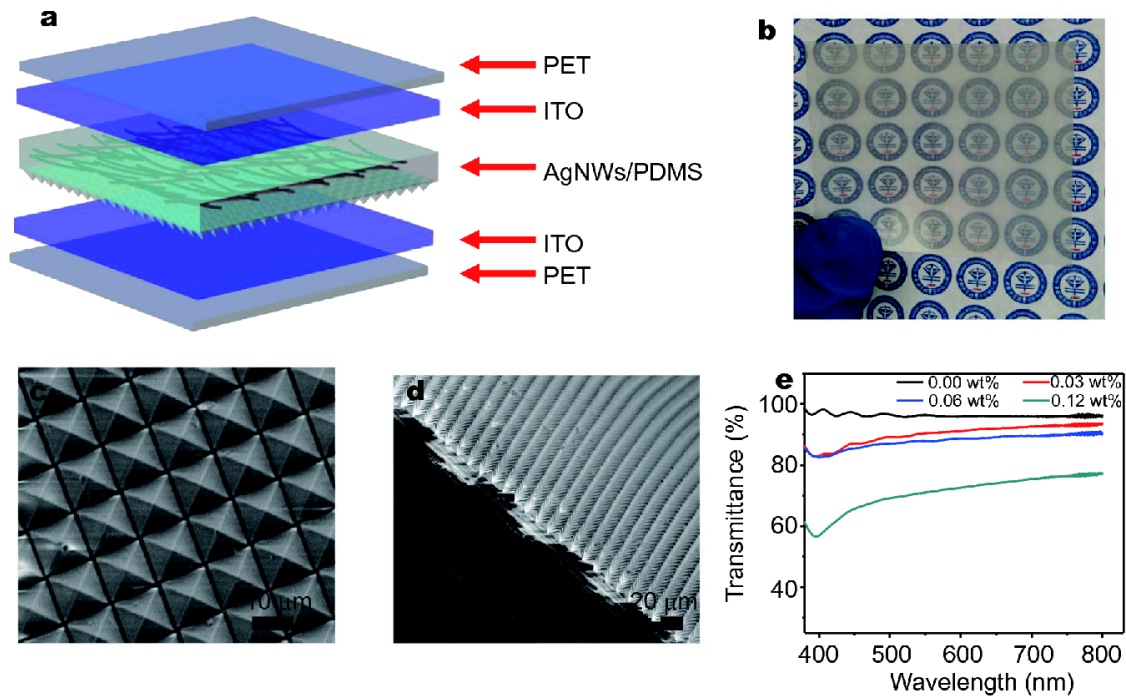

Figure 2 (a) Architecture of the flexible capacitive pressure sensor. (b) The photo image of a single pressure sensor device. (c) Top and (d) tilted SEM images of the micro-structured AgNWs/PDMS film. (e) Optical transmittance of the AgNWs/PDMS films with various mixing ratios of AgNWs.

dielectric constant of the composite dielectric film, and the value of $\varepsilon_{\mathrm{a}}$ can be determined by the content of the filler material and the deformation of the dielectric under pressure. $f_{\mathrm{c}}, f$ and $\varepsilon_{\mathrm{d}}$ are the percolation threshold, the filling factor, and the conductivities of the dielectric phases, respectively. According to the Equations (2) and (3), the incorporation of AgNWs can change $\varepsilon_{\mathrm{a}}$ of PDMS dielectrics, and the change of $\varepsilon_{\mathrm{a}}$ will be maximized when the concentration of AgNWs reaches a certain content. Combined with the reduced distance $d$ caused by pressure, the sensitivity of the sensor can be further enhanced.

First, to optimize the doping ratio of AgNWs, the response performance of the pressure sensors has been characterized. Fig. $3 \mathrm{~b}$ shows the capacitance changes of the non-patterned sensors under pressure with various mixing ratios of AgNWs. The sensitivity $S$ of the pressure sensor can be defined as: $S=\left(\left(C-C_{0}\right) / C_{0}\right) / \Delta p$, (Equation 4), where $p$ is the applied pressure, $C$ and $C_{0}$ represent the capacitance with and without pressure. The sensitivity of the sensor based on the unstructured PDMS film without AgNWs was much lower than that with the AgNWs/ PDMS composite film. The results show that the sensor with $0.12 \mathrm{wt} \%$ AgNWs exhibits the highest sensitivity. In the pressure range of $1 \mathrm{kPa}$, the sensitivity of the AgNWs/ PDMS composite film $\left(0.059 \mathrm{kPa}^{-1}\right)$ is about five times higher than that of the pure PDMS film $\left(0.012 \mathrm{kPa}^{-1}\right)$. And thus, the doping ratio of $0.12 \mathrm{wt} \%$ was chosen as the optimal dielectric of the capacitive pressure sensor.

On this basis, in order to further improve the sensitivity of the sensor, the pyramid-shaped microstructure was then introduced to the composite film. Fig. 3c, d show the sensitivity curves of the pressure sensor based on different types of dielectric films. It can be seen clearly that the sensor based on microstructured AgNWs/PDMS dielectric films with $0.12 \mathrm{wt} \%$ AgNWs exhibits higher pressure sensitivity than that based on the non-patterned or microstructured PDMS dielectric layer without AgNWs. For the sensor with $0.12 \mathrm{wt} \%$ AgNWs, the sensitivity is as high as $0.831 \mathrm{kPa}^{-1}$ in the low pressure range. Although the value decreases $\left(0.063 \mathrm{kPa}^{-1}\right)$ as the pressure increases, it is also comparable to some reported capacitive pressure sensors $[11,14]$. The outstanding sensitivity of the sensor at low pressure range combined with wide sensing range make it possible to detect slight touch information. Similarly, the sensitivity of the sensor based on microstructured PDMS dielectric layer without AgNWs is about $0.218 \mathrm{kPa}^{-1}$ at low pressure and decreases to $0.035 \mathrm{kPa}^{-1}$ with increased pressure. Compared with the previous researches shown in Fig. 3e and Table $\mathrm{S} 1$, the as-prepared sensors have better performance such as high sensitivity and low detection limit. These results show that the sensing performance of the as-fabricated capacitive pressure sensor based on a conventional PDMS film is successfully enhanced by changing geometrical structures and additional factors such as dielectric constant $\varepsilon$.

According to the above results, here we just discuss the pressure sensor based on microstructured $0.12 \mathrm{wt} \%$ AgNWs/PDMS dielectric film. The reliable capacitance changes of the sensor under different pressures are shown 

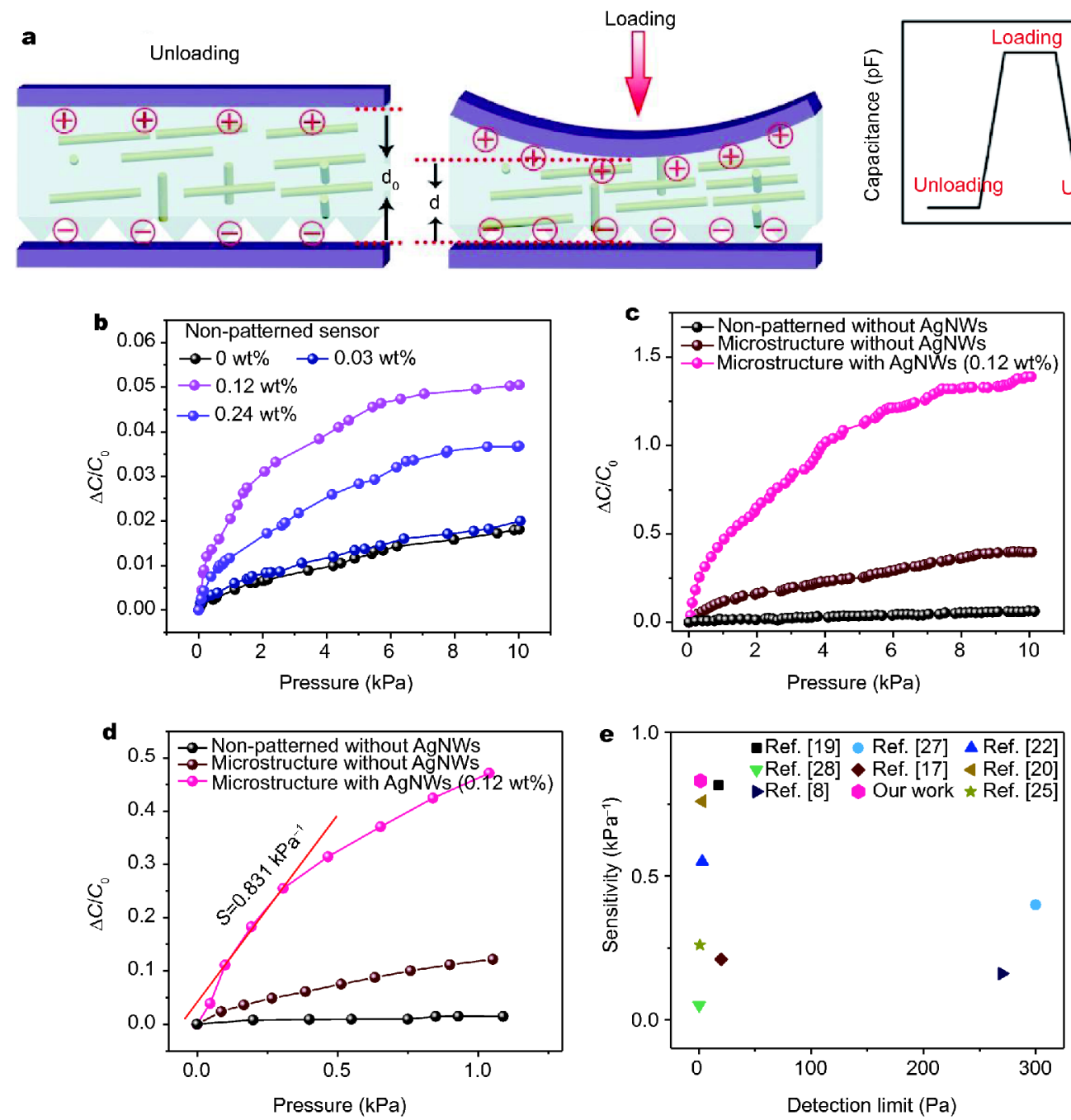

Figure 3 (a) Schematic illustration of the sensing mechanism of the capacitive pressure sensor. (b) Capacitance changes of the non-patterned sensors under pressure with various mixing ratios of AgNWs. (c, d) Sensitivity curves of the pressure sensor with different types of dielectric layer under applied pressure. The micro-structured sensor based on AgNWs/PDMS dielectric layer with $0.12 \mathrm{wt} \%$ AgNWs exhibits higher pressure sensitivity than that based on the non-patterned or micro-structured PDMS dielectric layer without AgNWs. (e) Comparison of the sensing performance of our work and previous research results $[8,17,19,20,22,25,27,28]$.

in Fig. 4a. It can be seen that the sensors exhibit a very stable response under dynamic pressure, which can be stably operated under static pressure and accurately measure the applied force. Fig. $4 \mathrm{~b}$ shows the real-time response curves of both types of microstructured sensors under pressures of $0.1 \mathrm{kPa}$ and $0.2 \mathrm{kPa}$, respectively. The results show that the microstructured pressure sensor with $0.12 \mathrm{wt} \%$ AgNWs exhibits a higher sensitivity and reproducibility than the sensors without AgNWs. Fig. 4c and insets show the fast response and relaxation times of the pressure sensor. When the pressure was loaded to the pressure sensor, the response time was less than $30 \mathrm{~ms}$, and a short relaxation time (less than $60 \mathrm{~ms}$ ) was ob- tained when the pressure was unloaded from the sensor. This pressure sensor based on AgNWs/PDMS composite film can also detect small changes in pressure. Due to the high sensitivity and fast reaction time of the pressure senor, some ultra-light objects such as a small piece of paper $(89 \mathrm{mg}, 1.4 \mathrm{~Pa})$, etc. loaded onto the sensor can induce the capacitance changes as shown in Fig. $4 \mathrm{~d}$. Durability and reliability are two key factors in the practical application of pressure sensors. Hence, the cyclic stability of the pressure sensor was then measured. Fig. 4e shows the capacitance change curves under a pressure of $1 \mathrm{kPa}$. It is worth noting that even after 10,000 cycles, the attenuation of the capacitance change can be negligible. 

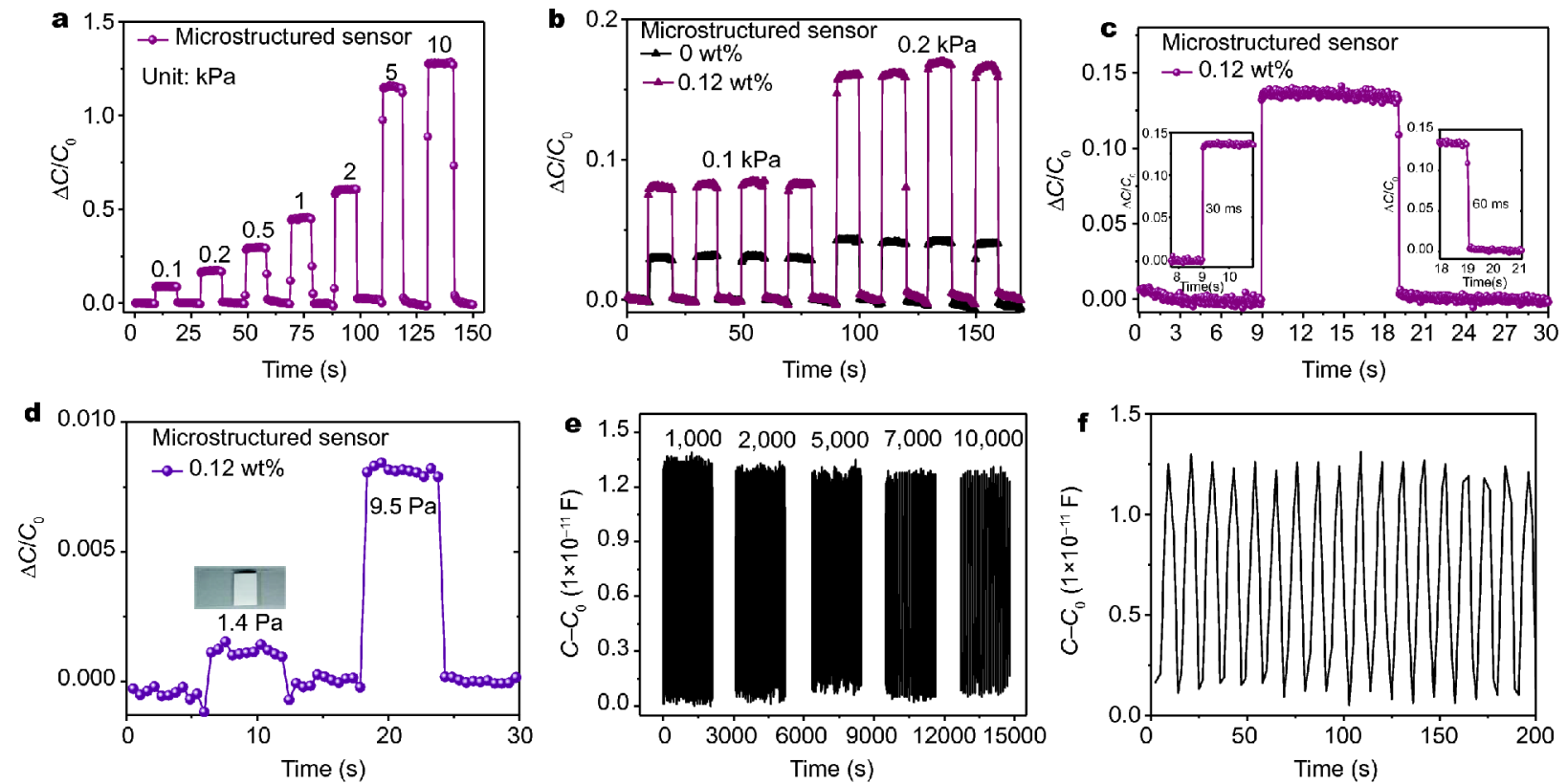

Figure 4 Characterization of capacitive pressure response of the sensor with the microstructured 0.12 wt\% AgNWs/PDMS dielectric layer. (a) Reliable capacitance change of the sensor under different pressures. (b) Repeated real-time response curves of both types of micro-structured sensors under pressures of $0.1 \mathrm{kPa}$ and $0.2 \mathrm{kPa}$, respectively. (c) Fast response time $(<30 \mathrm{~ms})$ and relaxation time of the sensor. (d) Transient response to the placing and removal of a small piece of paper, the first corresponding to a pressure of only 1.4 Pa. (e) Capacitance change curves recorded after 1,000, 2,000, 5,000, 7,000 and 10,000 cycles, respectively under a pressure of $1 \mathrm{kPa}$. (f) Magnified view of (e) after 7,000 loading-unloading cycles.

The entire 10,000 cycles are shown in Fig. S4. The large number of repetitive cycles confirms the excellent reproducibility of the sensor. As shown in the enlarged view (Fig. 4f), almost the same sharp peaks were observed in each cycle. The results show that the pressure sensors based on AgNWs/PDMS composite film have a long working life and stability.

In order to realize the practical application of flexible capacitive pressure sensor in human-computer interaction and electronic skin, it is necessary to fabricate a large-scale flexible pressure sensor array. Fig. 5a shows the schematic illustration of the final flexible $5 \times 5$ e-skin device and an enlarged pixel with a sandwich structure. By detecting the capacitance variation of each pixel, the array device can be used as a flexible wearable keyboard to implement the function of a conventional keyboard. The system-level block diagram in Fig. 5b shows the signal switching, conditioning, processing and wireless transmission paths from the signal conditioning of each pixel and output the corresponding conversion signal. The oscillating circuit is used to detect the input capacitance change of the array device. In addition, the microprocessor determines the key information by calculating and detecting the frequency of the oscillation circuit, and controls the input switching circuit to acquire the signals of the respective pixel points. Finally, the data is wirelessly transferred to the phone application via the WiFi module. Fig. $5 \mathrm{c}$ shows the smart keyboard that fits into the skin and mobile phone software. The flexible sensor array is able to achieve the function of keyboard. Demonstrations such as real-time inputting word "f" (Fig. 5d) and "flextronics" (Fig. 5e) are shown in the mobile application. Remarkably, such matrix of pressure sensor array reveals a great potential in the applications of human-computer interaction.

\section{CONCLUSIONS}

In summary, we used a simple and low-cost process to fabricate flexible, transparent and ultrasensitive capacitive pressure sensors by introducing patterned microstructured AgNWs/PMDS composite dielectric films. The as-fabricated capacitive pressure sensor has a large capacitive response $\left(0.831 \mathrm{kPa}^{-1}\right)$ when different pressures are applied, and can easily detect minute pressure $(1.4 \mathrm{~Pa})$. Moreover, the capacitive pressure sensor has a fast response $(<30 \mathrm{~ms})$ and relaxation $(<60 \mathrm{~ms})$ time. It also exhibits a high stability and durability after repeated cycles of more than 10,000 times. We also demonstrated the expansion of our sensors in the field of wearable electronic skin by making a $5 \times 5$ array of wearable flexible 

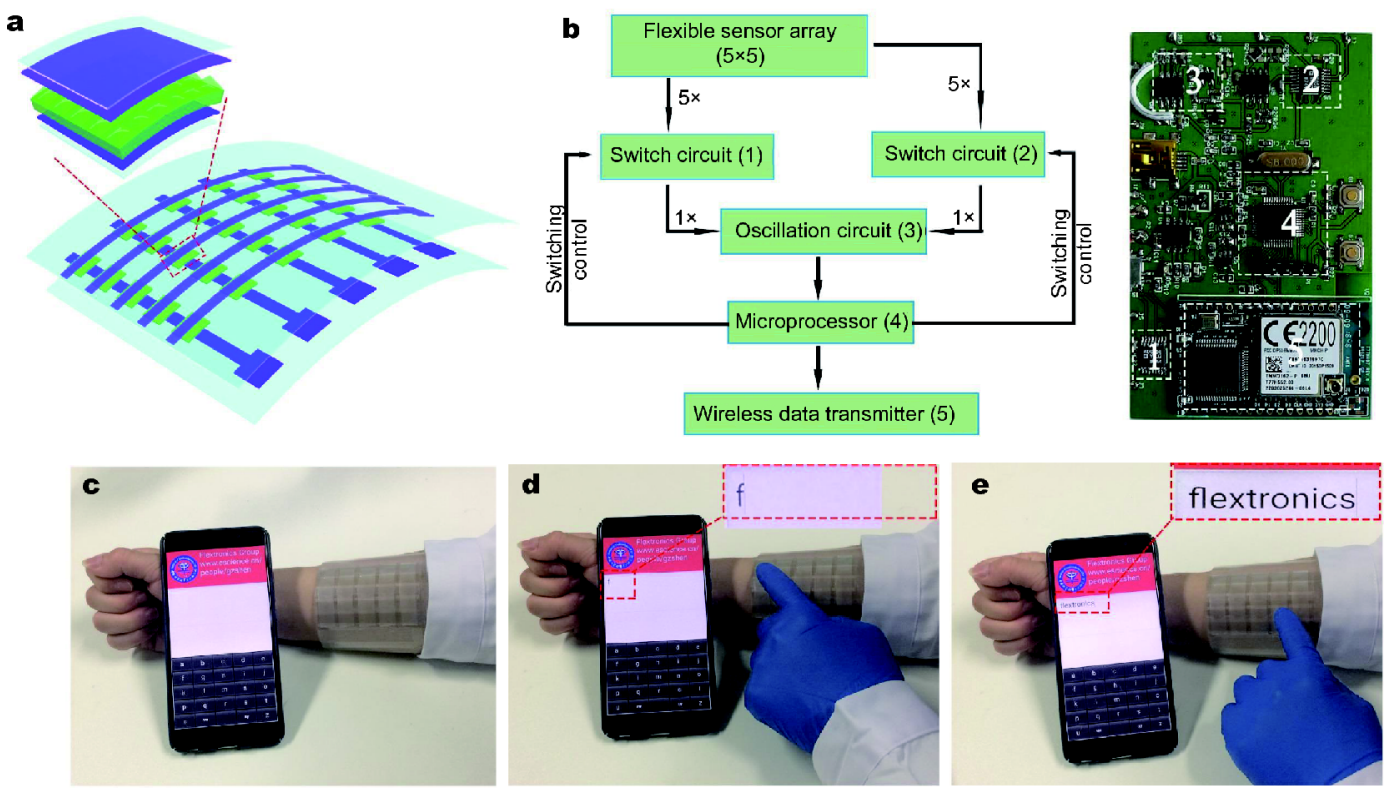

Figure 5 (a) Schematic illustration of the final flexible e-skin device and an enlarged pixel with a sandwich structure. (b) System-level block diagram of the wireless printed circuit board (PCB) showing the signal switching, conditioning, processing and wireless transmission paths from sensors to the mobile application (numbers in parentheses indicate the corresponding labelled components in the photo of the wireless PCB). The flexible sensor array is able to achieve the function of the keyboard (c). Demonstrations such as real-time inputting word "f" (d) and "flextronics" (e) are shown in the mobile application.

keyboards. The array-wearable keyboard shows a high sensitivity which can clearly distinguish the applied pressure to each pixel and implements a function similar to that of a conventional keyboard.

\section{Received 14 February 2018; accepted 28 March 2018;} published online 27 April 2018

1 Lou Z, Chen S, Wang L, et al. Ultrasensitive and ultraflexible eskins with dual functionalities for wearable electronics. Nano Energy, 2017, 38: 28-35

2 Khan Y, Ostfeld AE, Lochner CM, et al. Monitoring of vital signs with flexible and wearable medical devices. Adv Mater, 2016, 28: 4373-4395

3 Hammock ML, Chortos A, Tee BCK, et al. 25th anniversary article: the evolution of electronic skin (e-skin): a brief history, design considerations, and recent progress. Adv Mater, 2013, 25: 59976038

4 Wang L, Jackman JA, Tan EL, et al. High-performance, flexible electronic skin sensor incorporating natural microcapsule actuators. Nano Energy, 2017, 36: 38-45

5 Chen S, Lou Z, Chen D, et al. Polymer-enhanced highly stretchable conductive fiber strain sensor used for electronic data gloves. Adv Mater Technol, 2016, 1: 1600136

6 Lou Z, Chen S, Wang L, et al. An ultra-sensitive and rapid response speed graphene pressure sensors for electronic skin and health monitoring. Nano Energy, 2016, 23: 7-14

7 Kim CC, Lee $\mathrm{HH}$, Oh $\mathrm{KH}$, et al. Highly stretchable, transparent ionic touch panel. Science, 2016, 353: 682-687

8 Lee J, Kwon H, Seo J, et al. Conductive fiber-based ultrasensitive textile pressure sensor for wearable electronics. Adv Mater, 2015, 27: 2433-2439

9 Wang L, Jackman JA, Ng WB, et al. Flexible, graphene-coated biocomposite for highly sensitive, real-time molecular detection. Adv Funct Mater, 2016, 26: 8623-8630

10 Wang L, Chen D, Jiang K, et al. New insights and perspectives into biological materials for flexible electronics. Chem Soc Rev, 2017, 46: 6764-6815

11 Wang L, Jackman JA, Park JH, et al. A flexible, ultra-sensitive chemical sensor with 3D biomimetic templating for diabetes-related acetone detection. J Mater Chem B, 2017, 5: 4019-4024

12 Wang L, Ng WB, Jackman JA, et al. Graphene-functionalized natural microcapsules: modular building blocks for ultrahigh sensitivity bioelectronic platforms. Adv Funct Mater, 2016, 26: 2097-2103

13 Chou HH, Nguyen A, Chortos A, et al. A chameleon-inspired stretchable electronic skin with interactive colour changing controlled by tactile sensing. Nat Commun, 2015, 6: 8011

14 Pang C, Koo JH, Nguyen A, et al. Highly skin-conformal microhairy sensor for pulse signal amplification. Adv Mater, 2015, 27: 634-640

15 Lee BY, Kim J, Kim H, et al. Low-cost flexible pressure sensor based on dielectric elastomer film with micro-pores. Sensor Actuat A-Phys, 2016, 240: 103-109

16 Chen YS, Hsieh GW, Chen SP, et al. Zinc oxide nanowire-poly (methyl methacrylate) dielectric layers for polymer capacitive pressure sensors. ACS Appl Mater Interfaces, 2015, 7: 45-50

17 Kim SY, Park S, Park HW, et al. Highly sensitive and multimodal all-carbon skin sensors capable of simultaneously detecting tactile and biological stimuli. Adv Mater, 2015, 27: 4178-4185

18 Guo X, Huang Y, Cai X, et al. Capacitive wearable tactile sensor 
based on smart textile substrate with carbon black/silicone rubber composite dielectric. Meas Sci Technol, 2016, 27: 045105

19 Lee D, Lee H, Jeong Y, et al. Highly sensitive, transparent, and durable pressure sensors based on sea-urchin shaped metal nanoparticles. Adv Mater, 2016, 28: 9364-9369

20 Li T, Luo H, Qin L, et al. Flexible capacitive tactile sensor based on micropatterned dielectric layer. Small, 2016, 12: 5042-5048

21 Tee BCK, Chortos A, Dunn RR, et al. Tunable flexible pressure sensors using microstructured elastomer geometries for intuitive electronics. Adv Funct Mater, 2014, 24: 5427-5434

22 Mannsfeld SCB, Tee BCK, Stoltenberg RM, et al. Highly sensitive flexible pressure sensors with microstructured rubber dielectric layers. Nat Mater, 2010, 9: 859-864

23 Zhuo B, Chen S, Zhao M, et al. High sensitivity flexible capacitive pressure sensor using polydimethylsiloxane elastomer dielectric layer micro-structured by 3-D printed mold. IEEE J Electron Devices Soc, 2017, 5: 219-223

24 Mi Y, Chan Y, Trau D, et al. Micromolding of PDMS scaffolds and microwells for tissue culture and cell patterning: A new method of microfabrication by the self-assembled micropatterns of diblock copolymer micelles. Polymer, 2006, 47: 5124-5130

25 Chen S, Zhuo B, Guo X. Large area one-step facile processing of microstructured elastomeric dielectric film for high sensitivity and durable sensing over wide pressure range. ACS Appl Mater Interfaces, 2016, 8: 20364-20370

26 Schwartz G, Tee BCK, Mei J, et al. Flexible polymer transistors with high pressure sensitivity for application in electronic skin and health monitoring. Nat Commun, 2013, 4: 1859

27 Pan L, Chortos A, Yu G, et al. An ultra-sensitive resistive pressure sensor based on hollow-sphere microstructure induced elasticity in conducting polymer film. Nat Commun, 2014, 5: 3002
28

Boutry CM, Nguyen A, Lawal QO, et al. A sensitive and biodegradable pressure sensor array for cardiovascular monitoring. Adv Mater, 2015, 27: 6954-6961

29 Xu J, Wong M, Wong C. Super high dielectric constant carbon black-filled polymer composites as integral capacitor dielectrics. 54th Electronic Components and Technology Conference (IEEE Cat. No.04CH37546), 2004, 1: 536-541

30 Kirkpatrick S. The nature of percolation 'channels'. Solid State Commun, 1973, 12: 1279-1283

31 Zallen R. The formation of amorphous solids. In The Physics Of Amorphous Solids. New Jersey: Wiley-VCH, 1983, 1-32

32 Pecharromán C, Moya JS. Experimental evidence of a giant capacitance in insulator-conductor composites at the percolation threshold. Adv Mater, 2000, 12: 294-297

Acknowledgements This work was supported by the National Natural Science Foundation for Distinguished Young Scholars of China (NSFC, 61625404), the Key Research Program of Frontier Sciences, CAS (QYZDY-SSW-JWC004) and the NSFC (61504136).

Author contributions Shi $\mathrm{R}$ and Lou $\mathrm{Z}$ designed the devices and experiments; Shi R performed the experiments; Shi R, Lou Z and Chen S analyzed the data; Shi R and Chen S synthesized the Ag nanowires; Shi R wrote the paper with support from Lou Z and Chen S. All authors contributed to the general discussion.

Conflict of interest The authors declare no conflict of interest.

Supplementary information Supporting data are available in the online version of the paper. 

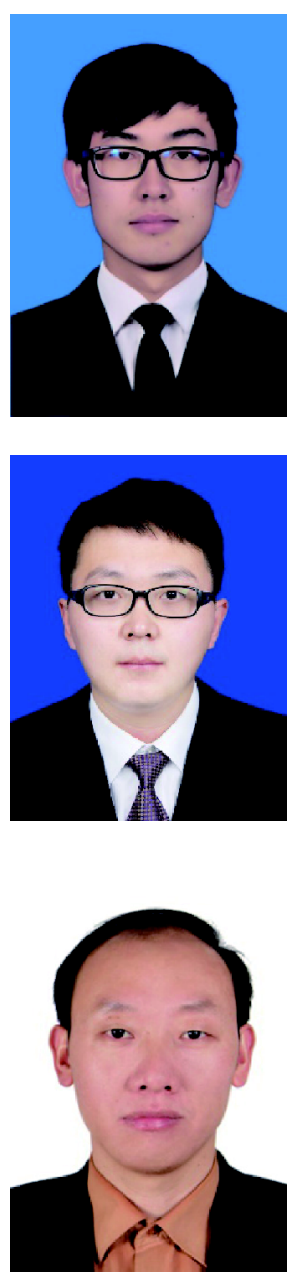

Ruilong Shi received his BSc degree from Jilin University in 2015. Now he is a graduate student at the Institute of Semiconductors, Chinese Academy of Sciences. His research interest focuses on flexible pressure sensor and wearable electronic devices.
Zheng Lou received his PhD degree from Jilin University in 2014. He joined the Institute of Semiconductors, Chinese Academy of Sciences as an Assistant Professor in 2014 and was promoted to Associate Professor in 2018. His current research focuses on flexible electronics, including pressure sensors, electronic-skin, transistors and photo-detectors.

Guozhen Shen received his BSc degree in 1999 from Anhui Normal University and PhD degree in 2003 from the University of Science and Technology of China. From 2004 to 2013, he conducted his research in Hanyang University (Korea), National Institute for Materials Science (Japan), University of Southern California (USA) and Huazhong University of Science and technology. He joined the Institute of Semiconductors, Chinese Academy of Sciences as a professor in 2013. His current research focuses on flexible electronics and printable electronics, including transistors, photodetectors, sensors and flexible energy-storage devices.

\title{
基于银纳米线/PDMS微结构复合电介质的柔性透明电容式压力传感器及其在穿戴式触摸键盘的应用
}

\author{
史瑞龙 ${ }^{1,2}$, 娄正 $^{1^{*}}$, 陈帅 ${ }^{1}$, 沈国震 ${ }^{1,2^{*}}$
}

摘要 对可穿戴电子设备来说, 采用简易制备技术得到具有高灵敏度、快速响应的压力传感器至关重要. 本文成功地研制了一种基于特 殊微结构银纳米线/PDMS复合电介质层材料的柔性透明电容式压力传感器. 与采用纯PDMS平面结构的电介质层器件相比, 有微结构的 传感器具有更高的灵敏度 $\left(0.831 \mathrm{kPa}^{-1},<0.5 \mathrm{kPa}\right)$, 更低的检测范围, 更好的稳定性和耐久性. 本文对导电填料含量和微结构的增强传感机 理也进行了讨论. 此外, 还研制了一个 $5 \times 5$ 的传感器阵列并用于柔性透明的可穿戴式触摸键盘系统. 研究结果表明所研制的压力传感器在 未来的电子皮肤领域将具有良好的应用前景. 